\title{
Oligoacenes. Theoretical Prediction of Open-Shell Singlet Diradical Ground States
}

\author{
Michael Bendikov*, K. N. Houk*, Hieu M. Duong, Kyle Starkey, Emily A. Carter* and
}

Fred Wudl*

Theoretical Methods

The GAUSSIAN $98^{1}$ series of programs was used for all $\mathrm{DFT}^{2}$ calculations. All molecules were fully optimized using the B3LYP, ${ }^{3}$ PW91, ${ }^{4}$ BPW91 ${ }^{4}$ and BLYP ${ }^{3}$ levels of theory with the $6-31 \mathrm{G}(\mathrm{d})$ basis set. The wavefunction for smaller oligoacenes up to pentacene is stable at B3LYP/6-31G(d). Full geometry optimization at CASSCF $(10,10) / 6-31 \mathrm{G}(\mathrm{d})$ was performed using GAUSSIAN $03^{5}$ using default guess for selecting active space orbitals and B3LYP/6-31G(d) optimized starting geometries. The five highest occupied molecular orbitals and the five lowest unoccupied molecular orbitals were included in the active space. Due to symmetry constraints $\left(D_{2 h}\right.$ symmetry for all oligoacenes) the active space remains constant during CAS(10,10) optimization and only $\square$-electrons were included in the active space.

Table S1. Calculated number of electrons outside closed-shell bonding orbitals (BO) and LUMO population from $\operatorname{CASSCF}(10 / 10) / 6-31 \mathrm{G}(\mathrm{d})$ calculations.

\begin{tabular}{l|cc} 
& $\begin{array}{c}\text { Total occupancy } \\
\text { outside BO }\end{array}$ & LUMO \\
\hline Anthracene & 0.386 & 0.131 \\
Tetracene & 0.376 & 0.123 \\
Pentacene & 0.420 & 0.119 \\
Hexacene & 0.477 & 0.217 \\
Heptacene & 0.545 & 0.239 \\
Octacene & 0.776 & 0.447
\end{tabular}

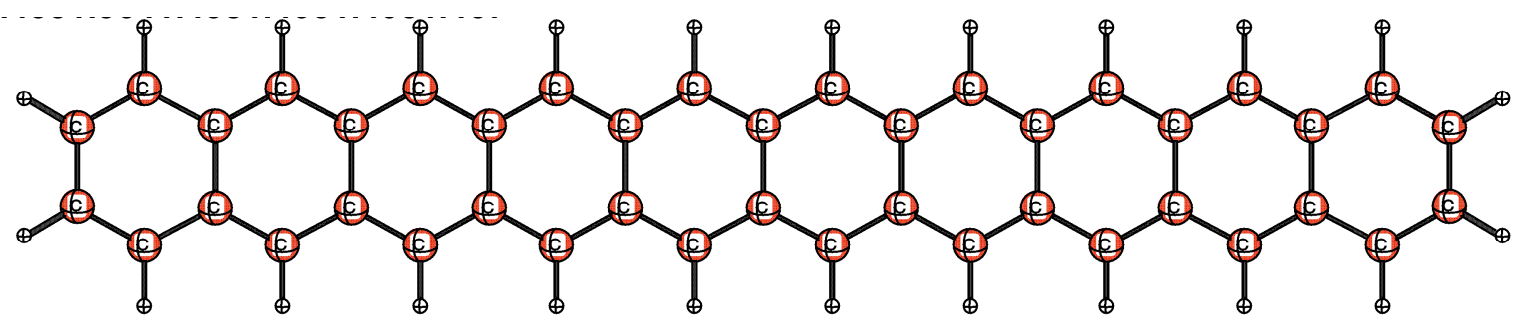

Figure S1. Calculated (RB3LYP/6-31G(d)) geometry of closed shell singlet decacene.

As an additional test for ability of DFT calculations to predict singlet-triplet splitting in extended $\square$ conjugated biradicals we calculated singlet-triplet splitting in triangulene, which is known to possess triplet ground state (Inoue, J.; Fukui, K.; Kubo, T.; Nakazawa, S.; Sato, K.; Shiomi, D.; Morita, Y.; Yamamoto, K.; Takui, T.; Nakasuji, K. J. Am. Chem. Soc. 2001, 123, 12702-12703). Indeed, triplet is below open shell singlet by $5 \mathrm{kcal} / \mathrm{mol}$ and below closed shell singlet by $25 \mathrm{kcal} / \mathrm{mol}$ at B3LYP/6-31G(d).

Despite the increase in reactivity as a result of benzannullation (from benzene to heptacene), it was predicted that the acene resonance energy per $\square$ electron remains nearly constant. ${ }^{6}$ Recently, the optical gap in nonacene $(n=9)$ and polyacene were predicted to be very large, $2.59 \mathrm{eV}$ and $2.46 \mathrm{eV}$, respectively, 
using density-matrix renormalization group calculations within a PPP Hamiltonian. ${ }^{7}$ In another recent paper, a singlet-triplet gap of $0.446 \mathrm{eV}$ was predicted for polyacenes on the basis of valence-bond (VB) calculations. ${ }^{8}$

${ }^{1}$ All calculations used the GAUSSIAN 98 series of programs. Gaussian 98, Revision A.11.4, Frisch, M. J.; Trucks, G. W.; Schlegel, H. B.; Scuseria, G. E.; Robb, M. A.; Cheeseman, J. R.; Zakrzewski, V. G.; Montgomery, Jr., J. A.; Stratmann, R. E.; Burant, J. C.; Dapprich, S.; Millam, J. M.; Daniels, A. D.; Kudin, K. N.; Strain, M. C.; Farkas, O.; Tomasi, J.; Barone, V.; Cossi, M.; Cammi, R.; Mennucci, B.; Pomelli, C.; Adamo, C.; Clifford, S.; Ochterski, J.; Petersson, G. A.; Ayala, P. Y.; Cui, Q.; Morokuma, K.; Rega, N.; Salvador, P.; Dannenberg, J. J.; Malick, D. K.; Rabuck, A. D.; Raghavachari, K.; Foresman, J. B.; Cioslowski, J.; Ortiz, J. V.; Baboul, A. G.; Stefanov, B. B.; Liu, G. Liashenko, A. Piskorz, P.; Komaromi, I.; Gomperts, R.; Martin, R. L.; Fox, D. J.; Keith, T.; AlLaham, M. A.; Peng, C. Y.; Nanayakkara, A.; Challacombe, M.; Gill, P. M. W.; Johnson, B.; Chen, W.; Wong, M. W.; Andres, J. L.; Gonzalez, C.; Head-Gordon, M.; Replogle, E. S.; and Pople, J. A. Gaussian, Inc., Pittsburgh PA, 2002.

2 (a) Parr, R. G.; Yang, W. Density-functional theory of atoms and molecules, Oxford University Press: New York, 1989. (b) Koch, W; Holthausen M. C. A Chemist's guide to density functional theory, Wiley-VCH: 2000.

${ }^{3}$ (a) Lee, C.; Yang, W.; Parr, R. G. Phys. Rev. B 1988, 37, 785. (b) Becke, A. D. J. Chem. Phys. 1993, 98, 5648.

${ }^{4}$ Perdew, J. P.; Burke, K.; Wang, Y. Phys. Rev. B 1996, 54, 16533. J. P. Perdew, J. A. Chevary, S. H. Vosko, K. A. Jackson, M. R. Pederson, D. J. Singh, C. Fiolhais, Phys. Rev. B 1992, 46, 6671-6687.

${ }^{5}$ Gaussian 03, Revision B.04, M. J. Frisch, G. W. Trucks, H. B. Schlegel, G. E. Scuseria, M. A. Robb, J. R. Cheeseman, J. A. Montgomery, Jr., T. Vreven, K. N. Kudin, J. C. Burant, J. M. Millam, S. S. Iyengar, J. Tomasi, V. Barone, B. Mennucci, M. Cossi, G. Scalmani, N. Rega, G. A. Petersson, H. Nakatsuji, M. Hada, M. Ehara, K. Toyota, R. Fukuda, J. Hasegawa, M. Ishida, T. Nakajima, Y. Honda, O. Kitao, H. Nakai, M. Klene, X. Li, J. E. Knox, H. P. Hratchian, J. B. Cross, C. Adamo, J. Jaramillo, R. Gomperts, R. E. Stratmann, O. Yazyev, A. J. Austin, R. Cammi, C. Pomelli, J. W. Ochterski, P. Y. Ayala, K. Morokuma, G. A. Voth, P. Salvador, J. J. Dannenberg, V. G. Zakrzewski, S. Dapprich, A. D. Daniels, M. C. Strain, O. Farkas, D. K. Malick, A. D. Rabuck, K. Raghavachari, J. B. Foresman, J. V. Ortiz, Q. Cui, A. G. Baboul, S. Clifford, J. Cioslowski, B. B. Stefanov, G. Liu, A. Liashenko, P. Piskorz, I. Komaromi, R. L. Martin, D. J. Fox, T. Keith, M. A. Al-Laham, C. Y. Peng, A. Nanayakkara, M. Challacombe, P. M. W. Gill, B. Johnson, W. Chen, M. W. Wong, C. Gonzalez, and J. A. Pople, Gaussian, Inc., Pittsburgh PA, 2003.

6 Schleyer, P. v. R.; Manoharan, M.; Jiao, H.; Stahl, F., Org. Lett. 2001, 3, 3643, For other studies on aromaticity of oligoacenes see: Krygowski, T. M.; Cyra_ski, M. K. Chem. Rev. 2001, 101, 1385 and references therein.

7 Raghu, C.; Anusooya Pati, Y.; Ramasesha, S. Phys. Rev. B 2002, 66, 035116/1-11.

8 Gao, Y.; Liu, C.-G.; Jiang, Y.-S. J. Phys. Chem. A 2002, 106, 2592-2597. 
Table S2. Absolute and zero-point energies.

\begin{tabular}{|c|c|c|c|}
\hline & B3LYP/6-31G* & ZPVE & 52 \\
\hline Hexacene & -1000.433488 & 209.62 & \\
\hline Hexacenebirad & -1000.433682 & 208.98 & 0.26 \\
\hline Hexacenetriplet & -1000.416257 & 208.27 & \\
\hline Heptacene & -1154.066724 & 238.75 & \\
\hline Heptacenebirad & -1154.069423 & 237.71 & $.8 C$ \\
\hline Heptacenetriplet & -1154.057771 & 237.52 & \\
\hline Octacene & -1307.699787 & 267.83 & \\
\hline Octacenebirad & -1307.706242 & 266.65 & 1.0 \\
\hline Octacenetriplet & -1307.697038 & 266.69 & \\
\hline Nonacene & -1461.332757 & 296.93 & \\
\hline Nonacenebirad & -1461.343237 & 295.64 & 1.26 \\
\hline Nonacenetriplet & -1461.334719 & 295.79 & \\
\hline Decacene & -1614.965671 & 326.00 & \\
\hline Decacenebirad & -1614.980131 & 324.65 & 1. \\
\hline \multirow[t]{2}{*}{ Decacenetripletopt } & -1614.971251 & 324.83 & \\
\hline & DFT/6-31G* & S2 & \\
\hline HeptaceneBLYP & -1153.568601 & 231.40Stable & \\
\hline HeptacenetripletBLYP & -1153.559174 & 230.63 & \\
\hline OctaceneBLYP & -1307.138664 & 259.59 & \\
\hline OctacenebiradBLYP & -1307.139700 & 258.97 & 0.67 \\
\hline NonaceneBLYP & -1460.708670 & 287.77 & \\
\hline NonacenebiradBLYP & -1460.712252 & 287.10 & $0 . \subseteq$ \\
\hline DecaceneBLYP & -1614.275718 & 315.71 & \\
\hline DecacenebiradBLYP & -1614.285261 & 315.32 & $1.1 \mathrm{C}$ \\
\hline DecacenetripletBLYP & -1614.283169 & 315.50 & \\
\hline HeptaceneBPW91 & -1153.933072 & 232.51 & \\
\hline HeptacenebiradBPW91 & -1153.933144 & 232.06 & 0.21 \\
\hline HeptacenePW91PW91 & -1153.629714 & 232.80 & \\
\hline HeptacenebiradPW91PW91 & -1153.629744 & 232.37 & $0.1 \approx$ \\
\hline
\end{tabular}

Table S3. Orbital energies.

\begin{tabular}{lrrrrr}
\multicolumn{5}{c}{ B3LYP/6-31G*//B3LYP/6-31G* } \\
HOMO & LUMC & HOMO $(\mathrm{eV})$ & LUMO $(\mathrm{eV})$ & gap \\
C6H6 & -0.24629 & 0.00362 & -6.70 & 0.10 & 6.80 \\
Naphtalene & -0.21269 & $-0.0352 \approx$ & -5.79 & -0.96 & 4.83 \\
Antracene & -0.19204 & -0.05996 & -5.23 & -1.63 & 3.59 \\
Tetracene & -0.17846 & -0.07632 & -4.86 & -2.08 & 2.78 \\
Pentacene & -0.169 & -0.08772 & -4.60 & -2.39 & 2.21 \\
Hexacene & -0.16214 & -0.096 & -4.41 & -2.61 & 1.80 \\
Hexacenebirad & -0.16382 & -0.09425 & -4.46 & -2.56 & 1.89 \\
Heptacene & -0.15694 & -0.10222 & -4.27 & -2.78 & 1.49 \\
Heptacenebirad & -0.16372 & -0.09554 & -4.45 & -2.60 & 1.86 \\
Octacene & -0.15294 & -0.10707 & -4.16 & -2.91 & 1.25 \\
Octacenebirad & -0.16373 & -0.09641 & -4.46 & -2.62 & 1.83 \\
Nonacene & -0.14992 & $-0.1107 \varepsilon$ & -4.08 & -3.01 & 1.06 \\
Nonacenebirad & -0.1639 & $-0.0969 \subseteq$ & -4.46 & -2.64 & 1.82 \\
Decacene & -0.14745 & -0.11382 & -4.01 & -3.10 & 0.92 \\
Decacenebirad & -0.16419 & -0.09732 & -4.47 & -2.65 & 1.82
\end{tabular}


Table S4. CASSCF(10/10)/6-31G(d) energies.

\begin{tabular}{|c|c|c|}
\hline & Singlet & $\square \mathrm{E}(\mathrm{S}-\mathrm{T})$ \\
\hline Benzene $(6,6)^{a}$ & $-230.7459147-230.59806$ & $92 . \varepsilon$ \\
\hline Antracene & $-536.1078017-536.03465$ & 45 \\
\hline Tetracene & $-688.743614 \quad-688.6731$ & 44.2 \\
\hline Pentacene & $-841.3916694-841.33651$ & \\
\hline Hexacene & $-994.0039042-993.98202$ & 13 \\
\hline Heptacene & $-1146.633926-1146.630^{k}$ & 2.5 \\
\hline Octacene & $-1299.285113-1299.2748$ & \\
\hline
\end{tabular}

a) $\operatorname{CASSCF}(6 / 6) / 6-31 \mathrm{G}(\mathrm{d})$

b) optimization not completely converged 
Table S5. Optimized geometries (at B3LYP/6-31G(d)).

Hexacene singlet RB3LYP

\begin{tabular}{|c|c|c|c|c|c|}
\hline $\begin{array}{l}\text { Center } \\
\text { Number }\end{array}$ & $\begin{array}{l}\text { Atomic } \\
\text { Number }\end{array}$ & $\begin{array}{r}\text { Atomic } \\
\text { Type }\end{array}$ & $\begin{array}{l}\text { Cooro } \\
\mathrm{X}\end{array}$ & $\begin{array}{c}\text { hates (Angs } \\
\text { Y }\end{array}$ & roms ) \\
\hline 1 & 6 & 0 & 0.000000 & 6.172241 & 1.411100 \\
\hline 2 & 6 & 0 & 0.000000 & 4.907691 & 0.728779 \\
\hline 3 & 1 & 0 & 0.000000 & 3.698587 & 2.496777 \\
\hline 4 & 6 & 0 & 0.000000 & 3.698818 & 1.408621 \\
\hline 5 & 6 & 0 & 0.000000 & 3.698818 & -1.408621 \\
\hline 6 & 6 & 0 & 0.000000 & 2.454528 & 0.730015 \\
\hline 7 & 6 & 0 & 0.000000 & 4.907691 & -0.728779 \\
\hline 8 & 6 & 0 & 0.000000 & 2.454528 & -0.730015 \\
\hline 9 & 6 & 0 & 0.000000 & 1.232441 & 1.409718 \\
\hline 10 & 6 & 0 & 0.000000 & 6.172241 & -1.411100 \\
\hline 11 & 1 & 0 & 0.000000 & 1.232439 & -2.497709 \\
\hline 12 & 1 & 0 & 0.000000 & 3.698587 & -2.496777 \\
\hline 13 & 6 & 0 & 0.000000 & 0.000000 & 0.730594 \\
\hline 14 & 1 & 0 & 0.000000 & 1.232439 & 2.497709 \\
\hline 15 & 6 & 0 & 0.000000 & -1.232441 & 1.409718 \\
\hline 16 & 6 & 0 & 0.000000 & 0.000000 & -0.730594 \\
\hline 17 & 1 & 0 & 0.000000 & -1.232439 & -2.497709 \\
\hline 18 & 6 & 0 & 0.000000 & 1.232441 & -1.409718 \\
\hline 19 & 6 & 0 & 0.000000 & -2.454528 & 0.730015 \\
\hline 20 & 1 & 0 & 0.000000 & -1.232439 & 2.497709 \\
\hline 21 & 6 & 0 & 0.000000 & -3.698818 & 1.408621 \\
\hline 22 & 6 & 0 & 0.000000 & -2.454528 & -0.730015 \\
\hline 23 & 6 & 0 & 0.000000 & -3.698818 & -1.408621 \\
\hline 24 & 6 & 0 & 0.000000 & -1.232441 & -1.409718 \\
\hline 25 & 6 & 0 & 0.000000 & 7.347388 & 0.717297 \\
\hline 26 & 1 & 0 & 0.000000 & 6.169798 & 2.498552 \\
\hline 27 & 6 & 0 & 0.000000 & 7.347388 & -0.717297 \\
\hline 28 & 1 & 0 & 0.000000 & 6.169798 & -2.498552 \\
\hline 29 & 1 & 0 & 0.000000 & 8.295785 & 1.247528 \\
\hline 30 & 1 & 0 & 0.000000 & 8.295785 & -1.247528 \\
\hline 31 & 6 & 0 & 0.000000 & -4.907691 & 0.728779 \\
\hline 32 & 1 & 0 & 0.000000 & -3.698587 & 2.496777 \\
\hline 33 & 6 & 0 & 0.000000 & -4.907691 & -0.728779 \\
\hline 34 & 1 & 0 & 0.000000 & -3.698587 & -2.496777 \\
\hline 35 & 6 & 0 & 0.000000 & -6.172241 & -1.411100 \\
\hline 36 & 6 & 0 & 0.000000 & -6.172241 & 1.411100 \\
\hline 37 & 1 & 0 & 0.000000 & -6.169798 & 2.498552 \\
\hline 38 & 6 & 0 & 0.000000 & -7.347388 & 0.717297 \\
\hline 39 & 1 & 0 & 0.000000 & -6.169798 & -2.498552 \\
\hline 40 & 6 & 0 & 0.000000 & -7.347388 & -0.717297 \\
\hline 41 & 1 & 0 & 0.000000 & -8.295785 & 1.247528 \\
\hline 42 & 1 & 0 & 0.000000 & -8.295785 & -1.247528 \\
\hline
\end{tabular}

\section{Hexacene singlet UB3LYP}

\begin{tabular}{cccccc} 
Center & Atomic & Atomic & \multicolumn{2}{c}{ Coordinates (Angstroms) } \\
Number & Number & Type & X & Y & Z \\
-1 & 6 & 0 & -6.172761 & -1.409953 & 0.000003 \\
2 & 6 & 0 & -4.911251 & -0.727182 & 0.000000 \\
3 & 1 & 0 & -3.698304 & -2.496217 & -0.000001 \\
4 & 6 & 0 & -3.698388 & -1.408034 & -0.000001 \\
5 & 6 & 0 & -3.698388 & 1.408034 & -0.000002
\end{tabular}




$\begin{array}{rl}6 & 6 \\ 7 & 6 \\ 8 & 6 \\ 9 & 6 \\ 10 & 6 \\ 11 & 1 \\ 12 & 1 \\ 13 & 6 \\ 14 & 1 \\ 15 & 6 \\ 16 & 6 \\ 17 & 1 \\ 18 & 6 \\ 19 & 6 \\ 20 & 1 \\ 21 & 6 \\ 22 & 6 \\ 23 & 6 \\ 24 & 6 \\ 25 & 6 \\ 26 & 1 \\ 27 & 6 \\ 28 & 1 \\ 29 & 1 \\ 30 & 1 \\ 31 & 6 \\ 32 & 1 \\ 33 & 6 \\ 34 & 1 \\ 35 & 6 \\ 36 & 6 \\ 37 & 1 \\ 38 & 6 \\ 39 & 1 \\ 40 & 1 \\ 41 & 1 \\ 42 & \end{array}$

$\begin{array}{rr}-2.457409 & -0.729266 \\ -4.911251 & 0.727182 \\ -2.457409 & 0.729265 \\ -1.232086 & -1.409801 \\ -6.172761 & 1.409953 \\ -1.232208 & 2.497827 \\ -3.698304 & 2.496217 \\ 0.000000 & -0.730433 \\ -1.232208 & -2.497827 \\ 1.232086 & -1.409801 \\ 0.000000 & 0.730432 \\ 1.232208 & 2.497826 \\ -1.232086 & 1.409801 \\ 2.457409 & -0.729266 \\ 1.232208 & -2.497827 \\ 3.698388 & -1.408034 \\ 2.457409 & 0.729265 \\ 3.698388 & 1.408034 \\ 1.232086 & 1.409801 \\ -7.350210 & -0.715856 \\ -6.170400 & -2.497471 \\ -7.350209 & 0.715857 \\ -6.170399 & 2.497471 \\ -8.297961 & -1.247336 \\ -8.297961 & 1.247337 \\ 4.911251 & -0.727182 \\ 3.698305 & -2.496217 \\ 4.911251 & 0.727182 \\ 3.698304 & 2.496217 \\ 6.172761 & 1.409953 \\ 6.172761 & -1.409953 \\ 6.170400 & -2.497471 \\ 7.350209 & -0.715856 \\ 6.170399 & 2.497471 \\ 7.350209 & 0.715857 \\ 8.297961 & -1.247336 \\ 8.297960 & 1.247337\end{array}$

$-0.000003$

$-0.000003$

$-0.000004$

0.000003

$-0.000004$

$-0.000001$

$-0.000004$

$-0.000004$

$-0.000004$

$-0.000004$

$-0.000004$

$-0.000003$

$-0.000003$

$-0.000004$

$-0.000002$

$-0.000002$

$-0.000001$

$-0.000003$

0.000004

0.000003

0.000004

0.000004

0.000006

0.000006

0.000001

$-0.000002$

0.000001

$-0.000001$

0.000004

0.000003

0.000002

0.000005

0.000004

0.000006

0.000007

0.000008

\section{Hexacene triplet}

\begin{tabular}{|c|c|c|c|c|c|}
\hline $\begin{array}{l}\text { Center } \\
\text { Number }\end{array}$ & $\begin{array}{l}\text { Atomic } \\
\text { Number }\end{array}$ & $\begin{array}{c}\text { Atomic } \\
\text { Type }\end{array}$ & $\begin{array}{l}\text { Coor } \\
\mathrm{X}\end{array}$ & $\begin{array}{r}\text { nates (Angs } \\
\text { Y }\end{array}$ & roms ) \\
\hline 1 & 6 & 0 & .000000 & 6.180773 & 1.402469 \\
\hline 2 & 6 & 0 & .000000 & 4.937965 & .719022 \\
\hline 3 & 1 & 0 & .000000 & 3.699705 & 2.491006 \\
\hline 4 & 6 & 0 & .000000 & 3.697114 & 1.402787 \\
\hline 5 & 6 & 0 & .000000 & 3.697114 & -1.402787 \\
\hline 6 & 6 & 0 & .000000 & 2.479120 & .727429 \\
\hline 7 & 6 & 0 & .000000 & 4.937965 & -.719022 \\
\hline 8 & 6 & 0 & .000000 & 2.479120 & -.727429 \\
\hline 9 & 6 & 0 & .000000 & 1.229190 & 1.408097 \\
\hline 10 & 6 & 0 & .000000 & 6.180773 & -1.402469 \\
\hline 11 & 1 & 0 & .000000 & 1.231402 & -2.496055 \\
\hline 12 & 1 & 0 & .000000 & 3.699705 & -2.491006 \\
\hline 13 & 6 & 0 & .000000 & .000000 & .733741 \\
\hline 14 & 1 & 0 & .000000 & 1.231402 & 2.496055 \\
\hline 15 & 6 & 0 & .000000 & -1.229190 & 1.408097 \\
\hline 16 & 6 & 0 & .000000 & .000000 & -.733741 \\
\hline 17 & 1 & 0 & .000000 & -1.231402 & -2.496055 \\
\hline 18 & 6 & 0 & .000000 & 1.229190 & -1.408097 \\
\hline 19 & 6 & 0 & .000000 & -2.479120 & .727429 \\
\hline
\end{tabular}




$\begin{array}{rrrrrr}20 & 1 & 0 & .000000 & -1.231402 & 2.496055 \\ 21 & 6 & 0 & .000000 & -3.697114 & 1.402787 \\ 22 & 6 & 0 & .000000 & -2.479120 & -.727429 \\ 23 & 6 & 0 & .000000 & -3.697114 & -1.402787 \\ 24 & 6 & 0 & .000000 & -1.229190 & -1.408097 \\ 25 & 6 & 0 & .000000 & 7.373183 & .707038 \\ 26 & 1 & 0 & .000000 & 6.178880 & 2.490034 \\ 27 & 6 & 0 & .00000 & 7.373183 & -.707038 \\ 28 & 1 & 0 & .000000 & 6.178880 & -2.490034 \\ 29 & 1 & 0 & .000000 & 8.316862 & 1.245637 \\ 30 & 1 & 0 & .000000 & 8.316862 & -1.245637 \\ 31 & 6 & 0 & .000000 & -4.937965 & .719022 \\ 32 & 1 & 0 & .000000 & -3.699705 & 2.491006 \\ 33 & 6 & 0 & .000000 & -4.937965 & -.719022 \\ 34 & 1 & 0 & .000000 & -3.699705 & -2.491006 \\ 35 & 6 & 0 & .000000 & -6.180773 & -1.402469 \\ 36 & 6 & 0 & .000000 & -6.180773 & 1.402469 \\ 37 & 1 & 0 & .000000 & -6.178880 & 2.490034 \\ 38 & 6 & 0 & .000000 & -7.373183 & .707038 \\ 39 & 1 & 0 & .000000 & -6.178880 & -2.490034 \\ 40 & 6 & 0 & .000000 & -7.373183 & -.707038 \\ 41 & 1 & 0 & .000000 & -8.316862 & 1.245637 \\ 42 & 1 & 000000 & -8.316862 & -1.245637\end{array}$

\section{Heptacene singlet RB3LYP}

\begin{tabular}{|c|c|c|c|c|c|}
\hline $\begin{array}{l}\text { Center } \\
\text { Number }\end{array}$ & $\begin{array}{l}\text { Atomic } \\
\text { Number }\end{array}$ & $\begin{array}{r}\text { Atomic } \\
\text { Type }\end{array}$ & $\begin{array}{l}\text { Coorc } \\
\mathrm{X}\end{array}$ & $\begin{array}{l}\text { nates (Ang } \\
\text { Y }\end{array}$ & roms ) \\
\hline 1 & 6 & 0 & 0.000000 & 7.401353 & 1.411503 \\
\hline 2 & 6 & 0 & 0.000000 & 6.136353 & 0.729157 \\
\hline 3 & 1 & 0 & 0.000000 & 4.929219 & 2.497472 \\
\hline 4 & 6 & 0 & 0.000000 & 4.928640 & 1.409302 \\
\hline 5 & 6 & 0 & 0.000000 & 4.928640 & -1.409302 \\
\hline 6 & 6 & 0 & 0.000000 & 3.682938 & 0.730720 \\
\hline 7 & 6 & 0 & 0.000000 & 6.136353 & -0.729157 \\
\hline 8 & 6 & 0 & 0.000000 & 3.682938 & -0.730720 \\
\hline 9 & 6 & 0 & 0.000000 & 2.463274 & 1.410479 \\
\hline 10 & 6 & 0 & 0.000000 & 7.401353 & -1.411503 \\
\hline 11 & 1 & 0 & 0.000000 & 2.463788 & -2.498527 \\
\hline 12 & 1 & 0 & 0.000000 & 4.929219 & -2.497472 \\
\hline 13 & 6 & 0 & 0.000000 & 1.227838 & 0.731696 \\
\hline 14 & 1 & 0 & 0.000000 & 2.463788 & 2.498527 \\
\hline 15 & 6 & 0 & 0.000000 & 0.000000 & 1.410801 \\
\hline 16 & 6 & 0 & 0.000000 & 1.227838 & -0.731696 \\
\hline 17 & 1 & 0 & 0.000000 & 0.000000 & -2.498833 \\
\hline 18 & 6 & 0 & 0.000000 & 2.463274 & -1.410479 \\
\hline 19 & 6 & 0 & 0.000000 & -1.227838 & 0.731696 \\
\hline 20 & 1 & 0 & 0.000000 & 0.000000 & 2.498833 \\
\hline 21 & 6 & 0 & 0.000000 & -2.463274 & 1.410479 \\
\hline 22 & 6 & 0 & 0.000000 & -1.227838 & -0.731696 \\
\hline 23 & 6 & 0 & 0.000000 & -2.463274 & -1.410479 \\
\hline 24 & 6 & 0 & 0.000000 & 0.000000 & -1.410801 \\
\hline 25 & 6 & 0 & 0.000000 & 8.576126 & 0.717462 \\
\hline 26 & 1 & 0 & 0.000000 & 7.399801 & 2.498980 \\
\hline 27 & 6 & 0 & 0.000000 & 8.576126 & -0.717462 \\
\hline 28 & 1 & 0 & 0.000000 & 7.399801 & -2.498980 \\
\hline 29 & 1 & 0 & 0.000000 & 9.524343 & 1.248118 \\
\hline 30 & 1 & 0 & 0.000000 & 9.524343 & -1.248118 \\
\hline 31 & 6 & 0 & 0.000000 & -3.682938 & 0.730720 \\
\hline 32 & 1 & 0 & 0.000000 & -2.463788 & 2.498527 \\
\hline 33 & 6 & 0 & 0.000000 & -3.682938 & -0.730720 \\
\hline
\end{tabular}




$\begin{array}{rrrrrr}34 & 1 & 0 & 0.000000 & -2.463788 & -2.498527 \\ 35 & 6 & 0 & 0.000000 & -4.928640 & -1.409302 \\ 36 & 6 & 0 & 0.000000 & -4.928640 & 1.409302 \\ 37 & 1 & 0 & 0.000000 & -4.929219 & 2.497472 \\ 38 & 6 & 0 & 0.000000 & -6.136353 & 0.729157 \\ 39 & 1 & 0 & 0.000000 & -4.929219 & -2.497472 \\ 40 & 6 & 0 & 0.000000 & -6.136353 & -0.729157 \\ 41 & 6 & 0 & 0.000000 & -7.401353 & 1.411503 \\ 42 & 6 & 0 & 0.000000 & -7.401353 & -1.411503 \\ 43 & 1 & 0 & 0.000000 & -7.399801 & 2.498980 \\ 44 & 6 & 0 & 0.000000 & -8.576126 & 0.717462 \\ 45 & 1 & 0 & 0.000000 & -7.399801 & -2.498980 \\ 46 & 6 & 0 & 0.000000 & -8.576126 & -0.717462 \\ 47 & 1 & 0 & 0.000000 & -9.524343 & 1.248118 \\ 48 & 1 & 0 & 0.000000 & -9.524343 & -1.248118\end{array}$

\section{Heptacene singlet UB3LYP}

\begin{tabular}{|c|c|c|c|c|c|}
\hline $\begin{array}{l}\text { Center } \\
\text { Number }\end{array}$ & $\begin{array}{l}\text { Atomic } \\
\text { Number }\end{array}$ & $\begin{array}{r}\text { Atomic } \\
\text { Type }\end{array}$ & $\begin{array}{l}\text { Coorc } \\
\mathrm{X}\end{array}$ & $\begin{array}{cc}\text { ates } & \text { (Ang } \\
& y\end{array}$ & roms ) \\
\hline 1 & 6 & 0 & 7.406687 & 0.000000 & 1.407844 \\
\hline 2 & 6 & 0 & 6.150249 & 0.000000 & 0.724972 \\
\hline 3 & 1 & 0 & 4.931256 & 0.000000 & 2.494725 \\
\hline 4 & 6 & 0 & 4.929863 & 0.000000 & 1.406477 \\
\hline 5 & 6 & 0 & 4.929863 & 0.000000 & -1.406477 \\
\hline 6 & 6 & 0 & 3.695586 & 0.000000 & 0.728645 \\
\hline 7 & 6 & 0 & 6.150249 & 0.000000 & -0.724972 \\
\hline 8 & 6 & 0 & 3.695586 & 0.000000 & -0.728645 \\
\hline 9 & 6 & 0 & 2.462610 & 0.000000 & 1.409245 \\
\hline 10 & 6 & 0 & 7.406687 & 0.000000 & -1.407844 \\
\hline 11 & 1 & 0 & 2.463992 & 0.000000 & -2.497308 \\
\hline 12 & 1 & 0 & 4.931256 & 0.000000 & -2.494725 \\
\hline 13 & 6 & 0 & 1.233051 & 0.000000 & 0.731630 \\
\hline 14 & 1 & 0 & 2.463992 & 0.000000 & 2.497308 \\
\hline 15 & 6 & 0 & 0.000000 & 0.000000 & 1.410299 \\
\hline 16 & 6 & 0 & 1.233051 & 0.000000 & -0.731630 \\
\hline 17 & 1 & 0 & 0.000000 & 0.000000 & -2.498320 \\
\hline 18 & 6 & 0 & 2.462610 & 0.000000 & -1.409245 \\
\hline 19 & 6 & 0 & -1.233051 & 0.000000 & 0.731630 \\
\hline 20 & 1 & 0 & 0.000000 & 0.000000 & 2.498320 \\
\hline 21 & 6 & 0 & -2.462610 & 0.000000 & 1.409245 \\
\hline 22 & 6 & 0 & -1.233051 & 0.000000 & -0.731630 \\
\hline 23 & 6 & 0 & -2.462610 & 0.000000 & -1.409245 \\
\hline 24 & 6 & 0 & 0.000000 & 0.000000 & -1.410299 \\
\hline 25 & 6 & 0 & 8.588108 & 0.000000 & 0.713453 \\
\hline 26 & 1 & 0 & 7.404516 & 0.000000 & 2.495387 \\
\hline 27 & 6 & 0 & 8.588108 & 0.000000 & -0.713453 \\
\hline 28 & 1 & 0 & 7.404516 & 0.000000 & -2.495387 \\
\hline 29 & 1 & 0 & 9.534592 & 0.000000 & 1.247151 \\
\hline 30 & 1 & 0 & 9.534592 & 0.000000 & -1.247151 \\
\hline 31 & 6 & 0 & -3.695586 & 0.000000 & 0.728645 \\
\hline 32 & 1 & 0 & -2.463992 & 0.000000 & 2.497308 \\
\hline 33 & 6 & 0 & -3.695586 & 0.000000 & -0.728645 \\
\hline 34 & 1 & 0 & -2.463992 & 0.000000 & -2.497308 \\
\hline 35 & 6 & 0 & -4.929863 & 0.000000 & -1.406477 \\
\hline 36 & 6 & 0 & -4.929863 & 0.000000 & 1.406477 \\
\hline 37 & 1 & 0 & -4.931256 & 0.000000 & 2.494725 \\
\hline 38 & 6 & 0 & -6.150249 & 0.000000 & 0.724972 \\
\hline 39 & 1 & 0 & -4.931256 & 0.000000 & -2.494725 \\
\hline 40 & 6 & 0 & -6.150249 & 0.000000 & -0.724972 \\
\hline 41 & 6 & 0 & -7.406687 & 0.000000 & 1.407844 \\
\hline
\end{tabular}




$\begin{array}{rrrrrr}42 & 6 & 0 & -7.406687 & 0.000000 & -1.407844 \\ 43 & 1 & 0 & -7.404516 & 0.000000 & 2.495387 \\ 44 & 6 & 0 & -8.588108 & 0.000000 & 0.713453 \\ 45 & 1 & 0 & -7.404516 & 0.000000 & -2.495387 \\ 46 & 6 & 0 & -8.588108 & 0.000000 & -0.713453 \\ 47 & 1 & 0 & -9.534592 & 0.000000 & 1.247151 \\ 48 & 1 & 0 & -9.534592 & 0.000000 & -1.247151\end{array}$

\section{Heptacene triplet}

\begin{tabular}{|c|c|c|c|c|c|}
\hline $\begin{array}{l}\text { Center } \\
\text { Number }\end{array}$ & $\begin{array}{l}\text { Atomic } \\
\text { Number }\end{array}$ & $\begin{array}{r}\text { Atomic } \\
\text { Type }\end{array}$ & $\begin{array}{l}\text { Coorc } \\
\mathrm{x}\end{array}$ & $\begin{array}{c}\text { nates (Ang } \\
Y\end{array}$ & roms ) \\
\hline 1 & 6 & 0 & 0.000000 & 7.411724 & 1.404398 \\
\hline 2 & 6 & 0 & 0.000000 & 6.163674 & 0.720631 \\
\hline 3 & 1 & 0 & 0.000000 & 4.931184 & 2.491467 \\
\hline 4 & 6 & 0 & 0.000000 & 4.929980 & 1.403229 \\
\hline 5 & 6 & 0 & 0.000000 & 4.929980 & -1.403229 \\
\hline 6 & 6 & 0 & 0.000000 & 3.709540 & 0.726107 \\
\hline 7 & 6 & 0 & 0.000000 & 6.163674 & -0.720631 \\
\hline 8 & 6 & 0 & 0.000000 & 3.709540 & -0.726107 \\
\hline 9 & 6 & 0 & 0.000000 & 2.459662 & 1.407340 \\
\hline 10 & 6 & 0 & 0.000000 & 7.411724 & -1.404398 \\
\hline 11 & 1 & 0 & 0.000000 & 2.461797 & -2.495373 \\
\hline 12 & 1 & 0 & 0.000000 & 4.931184 & -2.491467 \\
\hline 13 & 6 & 0 & 0.000000 & 1.239867 & 0.733120 \\
\hline 14 & 1 & 0 & 0.000000 & 2.461797 & 2.495373 \\
\hline 15 & 6 & 0 & 0.000000 & 0.000000 & 1.409688 \\
\hline 16 & 6 & 0 & 0.000000 & 1.239867 & -0.733120 \\
\hline 17 & 1 & 0 & 0.000000 & 0.000000 & -2.497609 \\
\hline 18 & 6 & 0 & 0.000000 & 2.459662 & -1.407340 \\
\hline 19 & 6 & 0 & 0.000000 & -1.239867 & 0.733120 \\
\hline 20 & 1 & 0 & 0.000000 & 0.000000 & 2.497609 \\
\hline 21 & 6 & 0 & 0.000000 & -2.459662 & 1.407340 \\
\hline 22 & 6 & 0 & 0.000000 & -1.239867 & -0.733120 \\
\hline 23 & 6 & 0 & 0.000000 & -2.459662 & -1.407340 \\
\hline 24 & 6 & 0 & 0.000000 & 0.000000 & -1.409688 \\
\hline 25 & 6 & 0 & 0.000000 & 8.599365 & 0.709635 \\
\hline 26 & 1 & 0 & 0.000000 & 7.409221 & 2.491941 \\
\hline 27 & 6 & 0 & 0.000000 & 8.599365 & -0.709635 \\
\hline 28 & 1 & 0 & 0.000000 & 7.409221 & -2.491941 \\
\hline 29 & 1 & 0 & 0.000000 & 9.544428 & 1.245846 \\
\hline 30 & 1 & 0 & 0.000000 & 9.544428 & -1.245846 \\
\hline 31 & 6 & 0 & 0.000000 & -3.709540 & 0.726107 \\
\hline 32 & 1 & 0 & 0.000000 & -2.461797 & 2.495373 \\
\hline 33 & 6 & 0 & 0.000000 & -3.709540 & -0.726107 \\
\hline 34 & 1 & 0 & 0.000000 & -2.461797 & -2.495373 \\
\hline 35 & 6 & 0 & 0.000000 & -4.929980 & -1.403229 \\
\hline 36 & 6 & 0 & 0.000000 & -4.929980 & 1.403229 \\
\hline 37 & 1 & 0 & 0.000000 & -4.931184 & 2.491467 \\
\hline 38 & 6 & 0 & 0.000000 & -6.163674 & 0.720631 \\
\hline 39 & 1 & 0 & 0.000000 & -4.931184 & -2.491467 \\
\hline 40 & 6 & 0 & 0.000000 & -6.163674 & -0.720631 \\
\hline 41 & 6 & 0 & 0.000000 & -7.411724 & 1.404398 \\
\hline 42 & 6 & 0 & 0.000000 & -7.411724 & -1.404398 \\
\hline 43 & 1 & 0 & 0.000000 & -7.409221 & 2.491941 \\
\hline 44 & 6 & 0 & 0.000000 & -8.599365 & 0.709635 \\
\hline 45 & 1 & 0 & 0.000000 & -7.409221 & -2.491941 \\
\hline 46 & 6 & 0 & 0.000000 & -8.599365 & -0.709635 \\
\hline 47 & 1 & 0 & 0.000000 & -9.544428 & 1.245846 \\
\hline 48 & 1 & 0 & 0.000000 & -9.544428 & -1.245846 \\
\hline
\end{tabular}




\section{Octacene singlet RB3LYP}

\begin{tabular}{|c|c|c|c|c|c|}
\hline $\begin{array}{l}\text { Center } \\
\text { Number }\end{array}$ & $\begin{array}{l}\text { Atomic } \\
\text { Number }\end{array}$ & $\begin{array}{r}\text { Atomic } \\
\text { Type }\end{array}$ & $\begin{array}{l}\text { Cooro } \\
x\end{array}$ & $\begin{array}{c}\text { inates (Angs } \\
\text { Y }\end{array}$ & ns ) \\
\hline 1 & 6 & 0 & 1.411680 & -8.631352 & .000000 \\
\hline 2 & 6 & 0 & .729393 & -7.366139 & .000000 \\
\hline 3 & 1 & 0 & 2.497729 & -6.159303 & .000000 \\
\hline 4 & 6 & 0 & 1.409564 & -6.158715 & .000000 \\
\hline 5 & 6 & 0 & -1.409568 & -6.158720 & .000000 \\
\hline 6 & 6 & 0 & .731096 & -4.912394 & .000000 \\
\hline 7 & 6 & 0 & -.729396 & -7.366140 & .000000 \\
\hline 8 & 6 & 0 & -.731094 & -4.912390 & .000000 \\
\hline 9 & 6 & 0 & 1.410974 & -3.693652 & .000000 \\
\hline 10 & 6 & 0 & -1.411692 & -8.631357 & .000000 \\
\hline 11 & 1 & 0 & -2.498992 & -3.694222 & .000000 \\
\hline 12 & 1 & 0 & -2.497732 & -6.159296 & .000000 \\
\hline 13 & 6 & 0 & .732306 & -2.456692 & .000000 \\
\hline 14 & 1 & 0 & 2.498990 & -3.694225 & .000000 \\
\hline 15 & 6 & 0 & 1.411557 & -1.231010 & .000000 \\
\hline 16 & 6 & 0 & -.732310 & -2.456687 & .000000 \\
\hline 17 & 1 & 0 & -2.499543 & -1.231184 & .000000 \\
\hline 18 & 6 & 0 & -1.410976 & -3.693657 & .000000 \\
\hline 19 & 6 & 0 & .732664 & -.000008 & .000000 \\
\hline 20 & 1 & 0 & 2.499539 & -1.231187 & .000000 \\
\hline 21 & 6 & 0 & 1.411559 & 1.231019 & .000000 \\
\hline 22 & 6 & 0 & -.732666 & -.000005 & .000000 \\
\hline 23 & 6 & 0 & -1.411559 & 1.231020 & .000000 \\
\hline 24 & 6 & 0 & -1.411560 & -1.231011 & .000000 \\
\hline 25 & 6 & 0 & .717585 & -9.806056 & .000000 \\
\hline 26 & 1 & 0 & 2.499160 & -8.629852 & .000000 \\
\hline 27 & 6 & 0 & -.717599 & -9.806060 & .000000 \\
\hline 28 & 1 & 0 & -2.499171 & -8.629842 & .000000 \\
\hline 29 & 1 & 0 & 1.248200 & -10.754301 & .000000 \\
\hline 30 & 1 & 0 & -1.248210 & -10.754306 & .000000 \\
\hline 31 & 6 & 0 & .732308 & 2.456675 & .000000 \\
\hline 32 & 1 & 0 & 2.499541 & 1.231189 & .000000 \\
\hline 33 & 6 & 0 & -.732306 & 2.456677 & .000000 \\
\hline 34 & 1 & 0 & -2.499541 & 1.231193 & .000000 \\
\hline 35 & 6 & 0 & -1.410976 & 3.693667 & .000000 \\
\hline 36 & 6 & 0 & 1.410980 & 3.693666 & .000000 \\
\hline 37 & 1 & 0 & 2.498995 & 3.694221 & .000000 \\
\hline 38 & 6 & 0 & .731096 & 4.912377 & .000000 \\
\hline 39 & 1 & 0 & -2.498992 & 3.694224 & .000000 \\
\hline 40 & 6 & 0 & -.731091 & 4.912378 & .000000 \\
\hline 41 & 6 & 0 & 1.409574 & 6.158731 & .000000 \\
\hline 42 & 6 & 0 & -1.409567 & 6.158732 & .000000 \\
\hline 43 & 1 & 0 & 2.497738 & 6.159286 & .000000 \\
\hline 44 & 6 & 0 & .729404 & 7.366130 & .000000 \\
\hline 45 & 1 & 0 & -2.497731 & 6.159288 & .000000 \\
\hline 46 & 6 & 0 & -.729396 & 7.366130 & .000000 \\
\hline 47 & 6 & 0 & 1.411696 & 8.631366 & .000000 \\
\hline 48 & 6 & 0 & -1.411687 & 8.631367 & .000000 \\
\hline 49 & 1 & 0 & 2.499176 & 8.629834 & .000000 \\
\hline 50 & 6 & 0 & .717609 & 9.806056 & .000000 \\
\hline 51 & 1 & 0 & -2.499167 & 8.629836 & .000000 \\
\hline 52 & 6 & 0 & -.717599 & 9.806056 & .000000 \\
\hline 53 & 1 & 0 & -1.248185 & 10.754316 & .000000 \\
\hline 54 & 1 & 0 & 1.248196 & 10.754315 & .000000 \\
\hline
\end{tabular}




\section{Octacene singlet UB3LYP}

\begin{tabular}{|c|c|c|c|c|c|}
\hline $\begin{array}{l}\text { Center } \\
\text { Number }\end{array}$ & $\begin{array}{l}\text { Atomic } \\
\text { Number }\end{array}$ & $\begin{array}{r}\text { Atomic } \\
\text { Type }\end{array}$ & $\begin{array}{l}\text { Coord } \\
X\end{array}$ & $\begin{array}{c}\text { nates (Angs } \\
\mathrm{Y}\end{array}$ & oms ) \\
\hline 1 & 6 & 0 & 8.640155 & 1.407481 & .000001 \\
\hline 2 & 6 & 0 & 7.384542 & .724221 & .000001 \\
\hline 3 & 1 & 0 & 6.162846 & 2.494138 & .000002 \\
\hline 4 & 6 & 0 & 6.162557 & 1.405954 & .000001 \\
\hline 5 & 6 & 0 & 6.162557 & -1.405954 & .000000 \\
\hline 6 & 6 & 0 & 4.930164 & .727584 & .000001 \\
\hline 7 & 6 & 0 & 7.384542 & -.724221 & .000000 \\
\hline 8 & 6 & 0 & 4.930164 & -.727585 & .000001 \\
\hline 9 & 6 & 0 & 3.694170 & 1.408667 & .000001 \\
\hline 10 & 6 & 0 & 8.640155 & -1.407481 & .000000 \\
\hline 11 & 1 & 0 & 3.694690 & -2.496665 & .000000 \\
\hline 12 & 1 & 0 & 6.162846 & -2.494139 & .000000 \\
\hline 13 & 6 & 0 & 2.467750 & .730745 & .000000 \\
\hline 14 & 1 & 0 & 3.694690 & 2.496665 & .000002 \\
\hline 15 & 6 & 0 & 1.230781 & 1.409987 & .000000 \\
\hline 16 & 6 & 0 & 2.467751 & -.730744 & .000000 \\
\hline 17 & 1 & 0 & 1.230934 & -2.497925 & .000000 \\
\hline 18 & 6 & 0 & 3.694170 & -1.408667 & .000000 \\
\hline 19 & 6 & 0 & .000000 & .731837 & -.000001 \\
\hline 20 & 1 & 0 & 1.230935 & 2.497925 & .000001 \\
\hline 21 & 6 & 0 & -1.230780 & 1.409987 & -.000001 \\
\hline 22 & 6 & 0 & .000000 & -.731837 & -.000001 \\
\hline 23 & 6 & 0 & -1.230780 & -1.409986 & -.000001 \\
\hline 24 & 6 & 0 & 1.230780 & -1.409986 & .000000 \\
\hline 25 & 6 & 0 & 9.822158 & .713114 & .000000 \\
\hline 26 & 1 & 0 & 8.637584 & 2.495002 & .000002 \\
\hline 27 & 6 & 0 & 9.822158 & -.713114 & .000000 \\
\hline 28 & 1 & 0 & 8.637585 & -2.495002 & .000000 \\
\hline 29 & 1 & 0 & 10.768781 & 1.246583 & .000000 \\
\hline 30 & 1 & 0 & 10.768780 & -1.246583 & -.000001 \\
\hline 31 & 6 & 0 & -2.467751 & .730745 & -.000002 \\
\hline 32 & 1 & 0 & -1.230933 & 2.497925 & -.000001 \\
\hline 33 & 6 & 0 & -2.467751 & -.730744 & -.000002 \\
\hline 34 & 1 & 0 & -1.230933 & -2.497925 & -.000001 \\
\hline 35 & 6 & 0 & -3.694169 & -1.408667 & -.000001 \\
\hline 36 & 6 & 0 & -3.694170 & 1.408667 & -.000002 \\
\hline 37 & 1 & 0 & -3.694690 & 2.496665 & -.000002 \\
\hline 38 & 6 & 0 & -4.930164 & .727585 & -.000002 \\
\hline 39 & 1 & 0 & -3.694689 & -2.496665 & -.000001 \\
\hline 40 & 6 & 0 & -4.930164 & -.727585 & -.000001 \\
\hline 41 & 6 & 0 & -6.162557 & 1.405954 & -.000001 \\
\hline 42 & 6 & 0 & -6.162556 & -1.405954 & .000000 \\
\hline 43 & 1 & 0 & -6.162847 & 2.494138 & -.000002 \\
\hline 44 & 6 & 0 & -7.384542 & .724221 & .000000 \\
\hline 45 & 1 & 0 & -6.162846 & -2.494138 & .000000 \\
\hline 46 & 6 & 0 & -7.384542 & -.724221 & .000000 \\
\hline 47 & 6 & 0 & -8.640155 & 1.407481 & .000000 \\
\hline 48 & 6 & 0 & -8.640154 & -1.407481 & .000002 \\
\hline 49 & 1 & 0 & -8.637586 & 2.495002 & .000000 \\
\hline 50 & 6 & 0 & -9.822159 & .713114 & .000002 \\
\hline 51 & 1 & 0 & -8.637585 & -2.495002 & .000003 \\
\hline 52 & 6 & 0 & -9.822159 & -.713114 & .000002 \\
\hline 53 & 1 & 0 & -10.768780 & -1.246584 & .000004 \\
\hline 54 & 1 & 0 & -10.768781 & 1.246583 & .000002 \\
\hline
\end{tabular}




\begin{tabular}{|c|c|c|c|c|c|}
\hline $\begin{array}{l}\text { Center } \\
\text { Number }\end{array}$ & $\begin{array}{l}\text { Atomic } \\
\text { Number }\end{array}$ & $\begin{array}{c}\text { Atomic } \\
\text { Type }\end{array}$ & $\begin{array}{l}\text { Coord } \\
\mathrm{X}\end{array}$ & $\begin{array}{c}\text { inates (Angs } \\
\mathrm{Y}\end{array}$ & roms) \\
\hline 1 & 6 & 0 & .000000 & 8.641154 & 1.405983 \\
\hline 2 & 6 & 0 & .000000 & 7.389122 & .722202 \\
\hline 3 & 1 & 0 & .000000 & 6.162015 & 2.492307 \\
\hline 4 & 6 & 0 & .000000 & 6.161471 & 1.404077 \\
\hline 5 & 6 & 0 & .000000 & 6.161471 & -1.404077 \\
\hline 6 & 6 & 0 & .000000 & 4.937243 & .725881 \\
\hline 7 & 6 & 0 & .000000 & 7.389122 & -.722202 \\
\hline 8 & 6 & 0 & .000000 & 4.937243 & -.725881 \\
\hline 9 & 6 & 0 & .000000 & 3.690787 & 1.407019 \\
\hline 10 & 6 & 0 & .000000 & 8.641154 & -1.405983 \\
\hline 11 & 1 & 0 & .000000 & 3.692291 & -2.495100 \\
\hline 12 & 1 & 0 & .000000 & 6.162015 & -2.492307 \\
\hline 13 & 6 & 0 & .000000 & 2.474676 & .731578 \\
\hline 14 & 1 & 0 & .000000 & 3.692291 & 2.495100 \\
\hline 15 & 6 & 0 & .000000 & 1.228502 & 1.409725 \\
\hline 16 & 6 & 0 & .000000 & 2.474676 & -.731578 \\
\hline 17 & 1 & 0 & .000000 & 1.229467 & -2.497691 \\
\hline 18 & 6 & 0 & .000000 & 3.690787 & -1.407019 \\
\hline 19 & 6 & 0 & .000000 & .000000 & .734917 \\
\hline 20 & 1 & 0 & .000000 & 1.229467 & 2.497691 \\
\hline 21 & 6 & 0 & .000000 & -1.228502 & 1.409725 \\
\hline 22 & 6 & 0 & .000000 & .000000 & -.734917 \\
\hline 23 & 6 & 0 & .000000 & -1.228502 & -1.409725 \\
\hline 24 & 6 & 0 & .000000 & 1.228502 & -1.409725 \\
\hline 25 & 6 & 0 & .000000 & 9.825364 & .711561 \\
\hline 26 & 1 & 0 & .000000 & 8.638551 & 2.493499 \\
\hline 27 & 6 & 0 & .000000 & 9.825364 & -.711561 \\
\hline 28 & 1 & 0 & .000000 & 8.638551 & -2.493499 \\
\hline 29 & 1 & 0 & .000000 & 10.771254 & 1.246288 \\
\hline 30 & 1 & 0 & .000000 & 10.771254 & -1.246288 \\
\hline 31 & 6 & 0 & .000000 & -2.474676 & .731578 \\
\hline 32 & 1 & 0 & .000000 & -1.229467 & 2.497691 \\
\hline 33 & 6 & 0 & .000000 & -2.474676 & -.731578 \\
\hline 34 & 1 & 0 & .000000 & -1.229467 & -2.497691 \\
\hline 35 & 6 & 0 & .000000 & -3.690787 & -1.407019 \\
\hline 36 & 6 & 0 & .000000 & -3.690787 & 1.407019 \\
\hline 37 & 1 & 0 & .000000 & -3.692291 & 2.495100 \\
\hline 38 & 6 & 0 & .000000 & -4.937243 & .725881 \\
\hline 39 & 1 & 0 & .000000 & -3.692291 & -2.495100 \\
\hline 40 & 6 & 0 & .000000 & -4.937243 & -.725881 \\
\hline 41 & 6 & 0 & .000000 & -6.161471 & 1.404077 \\
\hline 42 & 6 & 0 & .000000 & -6.161471 & -1.404077 \\
\hline 43 & 1 & 0 & .000000 & -6.162015 & 2.492307 \\
\hline 44 & 6 & 0 & .000000 & -7.389122 & .722202 \\
\hline 45 & 1 & 0 & .000000 & -6.162015 & -2.492307 \\
\hline 46 & 6 & 0 & .000000 & -7.389122 & -.722202 \\
\hline 47 & 6 & 0 & .000000 & -8.641154 & 1.405983 \\
\hline 48 & 6 & 0 & .000000 & -8.641154 & -1.405983 \\
\hline 49 & 1 & 0 & .000000 & -8.638551 & 2.493499 \\
\hline 50 & 6 & 0 & .000000 & -9.825364 & .711561 \\
\hline 51 & 1 & 0 & .000000 & -8.638551 & -2.493499 \\
\hline 52 & 6 & 0 & .000000 & -9.825364 & -.711561 \\
\hline 53 & 1 & 0 & .000000 & -10.771254 & -1.246288 \\
\hline 54 & 1 & 0 & .000000 & -10.771254 & 1.246288 \\
\hline
\end{tabular}

\section{Nonacene singlet RB3LYP}

$\begin{array}{ccr}\text { Center } & \text { Atomic } & \text { Atomic } \\ \text { Number } & \text { Number } & \text { Type }\end{array}$

Coordinates (Angstroms)

$\mathrm{X} Y \mathrm{Y}$ 


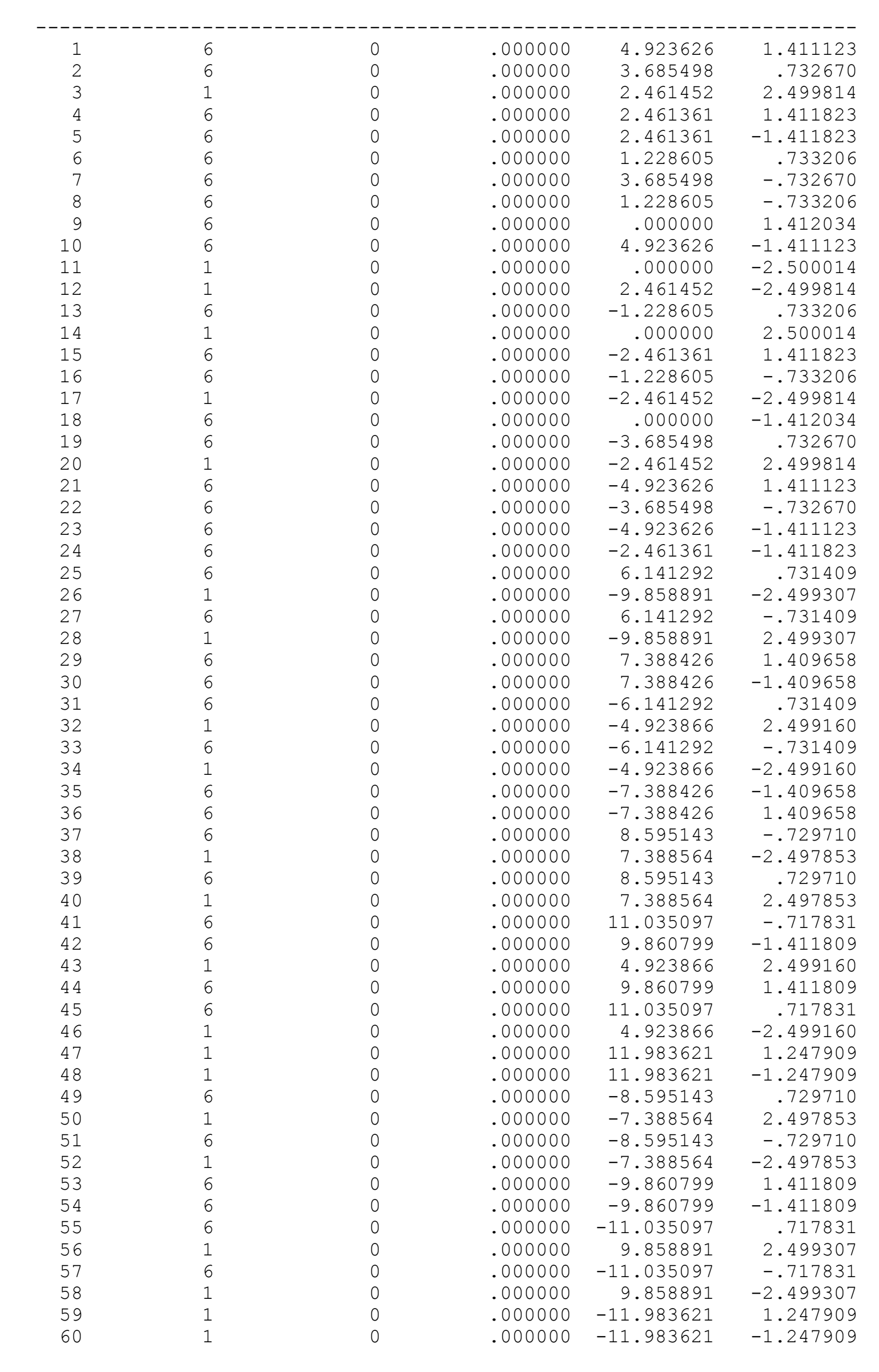




\section{Nonacene singlet UB3LYP}

$C, 0,-0.0000085836,4.9259496379,1.4083390756$ $\mathrm{C}, 0,-0.0000121441,3.7014976813,0.7303215999$ $\mathrm{H}, \mathrm{O},-0.0000158087,2.4619020821,2.4974973574$ $\mathrm{C}, 0,-0.0000153591,2.4618119634,1.409529963$ $\mathrm{C}, 0,-0.0000147607,2.4618120109,-1.4095301763$ $C, 0,-0.0000168868,1.2344316794,0.7316810523$ $\mathrm{C}, 0,-0.0000116591,3.7014977082,-0.7303218075$ $\mathrm{C}, 0,-0.0000167428,1.2344316966,-0.7316812899$ $\mathrm{C}, 0,-0.0000177751,-0.0000000026,1.4097667518$ $\mathrm{C}, 0,-0.0000074253,4.9259496949,-1.408339247$ $\mathrm{H}, \mathrm{O},-0.0000184077,0.0000000058,-2.4977256657$ $\mathrm{H}, \mathrm{0},-0.0000149375,2.4619021516,-2.4974975761$ $\mathrm{C}, 0,-0.00001702,-1.2344316784,0.73168107$ $\mathrm{H}, \mathrm{O},-0.0000180497,0.0000000112,2.4977254396$ $\mathrm{C}, 0,-0.0000152525,-2.4618119663,1.4095299982$ $\mathrm{C}, 0,-0.0000172616,-1.2344316799,-0.7316812763$ $\mathrm{H}, 0,-0.0000168159,-2.4619021389,-2.4974975511$ C, 0,-0.0000179123,0.0000000121,-1.4097669711 $C, 0,-0.0000120116,-3.7014976775,0.730321665$ $\mathrm{H}, \mathrm{O},-0.0000154082,-2.4619021108,2.4974973873$ C, $0,-0.0000075655,-4.9259496309,1.4083391788$ $\mathrm{C}, 0,-0.0000125792,-3.7014976918,-0.7303217469$ C, $0,-0.0000088749,-4.9259496867,-1.4083391532$ $\mathrm{C}, 0,-0.0000160535,-2.4618119903,-1.4095301498$ $\mathrm{C}, 0,-0.0000033201,6.1630140595,0.7272849882$ $\mathrm{H}, \mathrm{O}, 0.0000148163,-9.869913913,-2.4949741306$ C, $0,-0.0000025968,6.1630140939,-0.7272851205$ $\mathrm{H}, \mathrm{O}, 0.0000172943,-9.8699140111,2.4949743096$ $\mathrm{C}, 0,0.0000019694,7.3947947997,1.4058099833$ $\mathrm{C}, 0,0.0000035053,7.3947948853,-1.4058100418$ $\mathrm{C}, 0,-0.0000025317,-6.1630140639,0.7272851226$ $\mathrm{H}, \mathrm{O},-0.0000072859,-4.9263635734,2.4963536383$ $\mathrm{C}, 0,-0.000003283,-6.1630140724,-0.7272849936$ $\mathrm{H}, \mathrm{O},-0.0000096297,-4.926363767,-2.4963536164$ C, $0,0.0000021716,-7.3947948471,-1.4058099245$ $\mathrm{C}, 0,0.0000036572,-7.3947948315,1.4058101037$ $\mathrm{C}, 0,0.000009432,8.6169321183,-0.7241144024$ $\mathrm{H}, 0,0.0000040583,7.3950164278,-2.4939971092$ $\mathrm{C}, 0,0.0000085613,8.6169320698,0.7241144192$ $\mathrm{H}, 0,0.0000013448,7.3950162404,2.4939970485$ $\mathrm{C}, 0,0.0000231066,11.054460777,-0.7131002957$ $\mathrm{C}, 0,0.0000168116,9.8724971668,-1.4074562012$ $\mathrm{H}, \mathrm{O},-0.0000091912,4.9263636347,2.4963535321$ $\mathrm{C}, 0,0.0000149952,9.8724970142,1.4074563896$ $\mathrm{C}, 0,0.0000221645,11.0544607149,0.7131006498$ $\mathrm{H}, \mathrm{O},-0.0000071891,4.926363758,-2.4963537062$ $\mathrm{H}, \mathrm{0}, 0.0000273827,12.001082073,1.2465732955$ $\mathrm{H}, 0,0.0000291259,12.001082198,-1.2465728293$ $\mathrm{C}, 0,0.0000096718,-8.6169320988,0.7241145248$ $\mathrm{H}, 0,0.0000041374,-7.3950162957,2.4939971705$ $\mathrm{C}, 0,0.0000089211,-8.6169320872,-0.7241143053$ $\mathrm{H}, \mathrm{O}, 0.0000014779,-7.3950163742,-2.4939969959$ $\mathrm{C}, 0,0.0000167933,-9.8724971217,1.4074563879$ $\mathrm{C}, 0,0.0000154223,-9.8724970651,-1.4074562044$ $\mathrm{C}, 0,0.0000230758,-11.0544607593,0.7131005347$ $\mathrm{H}, \mathrm{O}, \mathrm{0} .0000142608,9.8699136803,2.4949743044$ $\mathrm{C}, 0,0.0000223932,-11.0544607377,-0.7131004099$ $\mathrm{H}, 0,0.0000175812,9.8699141075,-2.494974122$ $\mathrm{H}, 0,0.0000286816,-12.001082162,1.2465730962$ $\mathrm{H}, 0,0.0000275098,-12.0010821195,-1.246573018$ 


\section{Nonacene triplet}

\begin{tabular}{|c|c|c|c|c|c|}
\hline $\begin{array}{l}\text { Center } \\
\text { Number }\end{array}$ & $\begin{array}{l}\text { Atomic } \\
\text { Number }\end{array}$ & $\begin{array}{r}\text { Atomic } \\
\text { Type }\end{array}$ & $\begin{array}{l}\text { Coorc } \\
\mathrm{X}\end{array}$ & $\begin{array}{c}\text { inates (Ang } \\
\text { Y }\end{array}$ & (coms) \\
\hline 1 & 6 & 0 & .000000 & 4.922474 & 1.407063 \\
\hline 2 & 6 & 0 & .000000 & 3.706189 & .730462 \\
\hline 3 & 1 & 0 & .000000 & 2.459598 & 2.497293 \\
\hline 4 & 6 & 0 & .000000 & 2.458105 & 1.409240 \\
\hline 5 & 6 & 0 & .000000 & 2.458105 & -1.409240 \\
\hline 6 & 6 & 0 & .000000 & 1.237348 & .734625 \\
\hline 7 & 6 & 0 & .000000 & 3.706189 & -.730462 \\
\hline 8 & 6 & 0 & .000000 & 1.237348 & -.734625 \\
\hline 9 & 6 & 0 & .000000 & .000000 & 1.410401 \\
\hline 10 & 6 & 0 & .000000 & 4.922474 & -1.407063 \\
\hline 11 & 1 & 0 & .000000 & .000000 & -2.498417 \\
\hline 12 & 1 & 0 & .000000 & 2.459598 & -2.497293 \\
\hline 13 & 6 & 0 & .000000 & -1.237348 & .734625 \\
\hline 14 & 1 & 0 & .000000 & .000000 & 2.498417 \\
\hline 15 & 6 & 0 & .000000 & -2.458105 & 1.409240 \\
\hline 16 & 6 & 0 & .000000 & -1.237348 & -.734625 \\
\hline 17 & 1 & 0 & .000000 & -2.459598 & -2.497293 \\
\hline 18 & 6 & 0 & .000000 & .000000 & -1.410401 \\
\hline 19 & 6 & 0 & .000000 & -3.706189 & .730462 \\
\hline 20 & 1 & 0 & .000000 & -2.459598 & 2.497293 \\
\hline 21 & 6 & 0 & .000000 & -4.922474 & 1.407063 \\
\hline 22 & 6 & 0 & .000000 & -3.706189 & -.730462 \\
\hline 23 & 6 & 0 & .000000 & -4.922474 & -1.407063 \\
\hline 24 & 6 & 0 & .000000 & -2.458105 & -1.409240 \\
\hline 25 & 6 & 0 & .000000 & 6.164705 & .726239 \\
\hline 26 & 1 & 0 & .000000 & -9.868760 & -2.494715 \\
\hline 27 & 6 & 0 & .000000 & 6.164705 & -.726239 \\
\hline 28 & 1 & 0 & .000000 & -9.868760 & 2.494715 \\
\hline 29 & 6 & 0 & .000000 & 7.392818 & 1.404963 \\
\hline 30 & 6 & 0 & .000000 & 7.392818 & -1.404963 \\
\hline 31 & 6 & 0 & .000000 & -6.164705 & .726239 \\
\hline 32 & 1 & 0 & .000000 & -4.923917 & 2.495166 \\
\hline 33 & 6 & 0 & .000000 & -6.164705 & -.726239 \\
\hline 34 & 1 & 0 & .000000 & -4.923917 & -2.495166 \\
\hline 35 & 6 & 0 & .000000 & -7.392818 & -1.404963 \\
\hline 36 & 6 & 0 & .000000 & -7.392818 & 1.404963 \\
\hline 37 & 6 & 0 & .000000 & 8.616047 & -.723592 \\
\hline 38 & 1 & 0 & .000000 & 7.393453 & -2.493183 \\
\hline 39 & 6 & 0 & .000000 & 8.616047 & .723592 \\
\hline 40 & 1 & 0 & .000000 & 7.393453 & 2.493183 \\
\hline 41 & 6 & 0 & .000000 & 11.052999 & -.712889 \\
\hline 42 & 6 & 0 & .000000 & 9.871021 & -1.407202 \\
\hline 43 & 1 & 0 & .000000 & 4.923917 & 2.495166 \\
\hline 44 & 6 & 0 & .000000 & 9.871021 & 1.407202 \\
\hline 45 & 6 & 0 & .000000 & 11.052999 & .712889 \\
\hline 46 & 1 & 0 & .000000 & 4.923917 & -2.495166 \\
\hline 47 & 1 & 0 & .000000 & 11.999402 & 1.246761 \\
\hline 48 & 1 & 0 & .000000 & 11.999402 & -1.246761 \\
\hline 49 & 6 & 0 & .000000 & -8.616047 & .723592 \\
\hline 50 & 1 & 0 & .000000 & -7.393453 & 2.493183 \\
\hline 51 & 6 & 0 & .000000 & -8.616047 & -.723592 \\
\hline 52 & 1 & 0 & .000000 & -7.393453 & -2.493183 \\
\hline 53 & 6 & 0 & .000000 & -9.871021 & 1.407202 \\
\hline 54 & 6 & 0 & .000000 & -9.871021 & -1.407202 \\
\hline 55 & 6 & 0 & .000000 & -11.052999 & .712889 \\
\hline 56 & 1 & 0 & .000000 & 9.868760 & 2.494715 \\
\hline 57 & 6 & 0 & .000000 & -11.052999 & -.712889 \\
\hline 58 & 1 & 0 & .000000 & 9.868760 & -2.494715 \\
\hline
\end{tabular}


$\begin{array}{rrrrrr}59 & 1 & 0 & .000000 & -11.999402 & 1.246761 \\ 60 & 1 & 0 & 000000 & -11.999402 & -1.246761\end{array}$

\section{Decacene singlet RB3LYP}

\begin{tabular}{|c|c|c|c|c|c|}
\hline \multirow{2}{*}{$\begin{array}{l}\text { Center } \\
\text { Number }\end{array}$} & \multirow{2}{*}{$\begin{array}{l}\text { Atomic } \\
\text { Number }\end{array}$} & \multirow{2}{*}{$\begin{array}{r}\text { Atomic } \\
\text { Type }\end{array}$} & \multicolumn{3}{|c|}{ Coordinates (Angstroms) } \\
\hline & & & $\begin{array}{c}X \\
------1\end{array}$ & $\begin{array}{c}Y \\
---------.\end{array}$ & $\begin{array}{c}\mathrm{Z} \\
----------\end{array}$ \\
\hline 1 & 6 & 0 & 0.000000 & 11.090345 & 1.411798 \\
\hline 2 & 6 & 0 & 0.000000 & 9.824806 & 0.729719 \\
\hline 3 & 1 & 0 & 0.000000 & 8.618647 & 2.497987 \\
\hline 4 & 6 & 0 & 0.000000 & 8.618058 & 1.409785 \\
\hline 5 & 6 & 0 & 0.000000 & 8.618058 & -1.409785 \\
\hline 6 & 6 & 0 & 0.000000 & 7.370873 & 0.731625 \\
\hline 7 & 6 & 0 & 0.000000 & 9.824806 & -0.729719 \\
\hline 8 & 6 & 0 & 0.000000 & 7.370873 & -0.731625 \\
\hline 9 & 6 & 0 & 0.000000 & 6.153434 & 1.411329 \\
\hline 10 & 6 & 0 & 0.000000 & 11.090345 & -1.411798 \\
\hline 11 & 1 & 0 & 0.000000 & 6.153821 & -2.499396 \\
\hline 12 & 1 & 0 & 0.000000 & 8.618647 & -2.497987 \\
\hline 13 & 6 & 0 & 0.000000 & 4.914812 & 0.733006 \\
\hline 14 & 1 & 0 & 0.000000 & 6.153821 & 2.499396 \\
\hline 15 & 6 & 0 & 0.000000 & 3.691406 & 1.412091 \\
\hline 16 & 6 & 0 & 0.000000 & 4.914812 & -0.733006 \\
\hline 17 & 1 & 0 & 0.000000 & 3.691260 & -2.500129 \\
\hline 18 & 6 & 0 & 0.000000 & 6.153434 & -1.411329 \\
\hline 19 & 6 & 0 & 0.000000 & 2.457555 & 0.733547 \\
\hline 20 & 1 & 0 & 0.000000 & 3.691260 & 2.500129 \\
\hline 21 & 6 & 0 & 0.000000 & 1.230332 & 1.412386 \\
\hline 22 & 6 & 0 & 0.000000 & 2.457555 & -0.733547 \\
\hline 23 & 6 & 0 & 0.000000 & 1.230332 & -1.412386 \\
\hline 24 & 6 & 0 & 0.000000 & 3.691406 & -1.412091 \\
\hline 25 & 6 & 0 & 0.000000 & 12.264759 & 0.717717 \\
\hline 26 & 1 & 0 & 0.000000 & 11.088911 & 2.499305 \\
\hline 27 & 6 & 0 & 0.000000 & 12.264759 & -0.717717 \\
\hline 28 & 1 & 0 & 0.000000 & 11.088911 & -2.499305 \\
\hline 29 & 1 & 0 & 0.000000 & 13.213111 & 1.248177 \\
\hline 30 & 1 & 0 & 0.000000 & 13.213111 & -1.248177 \\
\hline 31 & 6 & 0 & 0.000000 & 0.000000 & 0.733618 \\
\hline 32 & 1 & 0 & 0.000000 & 1.230087 & 2.500407 \\
\hline 33 & 6 & 0 & 0.000000 & 0.000000 & -0.733618 \\
\hline 34 & 1 & 0 & 0.000000 & 1.230087 & -2.500407 \\
\hline 35 & 6 & 0 & 0.000000 & -1.230332 & -1.412386 \\
\hline 36 & 6 & 0 & 0.000000 & -1.230332 & 1.412386 \\
\hline 37 & 1 & 0 & 0.000000 & -1.230087 & 2.500407 \\
\hline 38 & 6 & 0 & 0.000000 & -2.457555 & 0.733547 \\
\hline 39 & 1 & 0 & 0.000000 & -1.230087 & -2.500407 \\
\hline 40 & 6 & 0 & 0.000000 & -2.457555 & -0.733547 \\
\hline 41 & 6 & 0 & 0.000000 & -3.691406 & 1.412091 \\
\hline 42 & 6 & 0 & 0.000000 & -3.691406 & -1.412091 \\
\hline 43 & 1 & 0 & 0.000000 & -3.691260 & 2.500129 \\
\hline 44 & 6 & 0 & 0.000000 & -4.914812 & 0.733006 \\
\hline 45 & 1 & 0 & 0.000000 & -3.691260 & -2.500129 \\
\hline 46 & 6 & 0 & 0.000000 & -4.914812 & -0.733006 \\
\hline 47 & 6 & 0 & 0.000000 & -6.153434 & 1.411329 \\
\hline 48 & 6 & 0 & 0.000000 & -6.153434 & -1.411329 \\
\hline 49 & 1 & 0 & 0.000000 & -6.153821 & 2.499396 \\
\hline 50 & 6 & 0 & 0.000000 & -7.370873 & 0.731625 \\
\hline 51 & 1 & 0 & 0.000000 & -6.153821 & -2.499396 \\
\hline 52 & 6 & 0 & 0.000000 & -7.370873 & -0.731625 \\
\hline 53 & 6 & 0 & 0.000000 & -8.618058 & -1.409785 \\
\hline 54 & 6 & 0 & 0.000000 & -8.618058 & 1.409785 \\
\hline
\end{tabular}




$\begin{array}{rrrrrr}55 & 6 & 0 & 0.000000 & -9.824806 & -0.729719 \\ 56 & 1 & 0 & 0.000000 & -8.618647 & -2.497987 \\ 57 & 6 & 0 & 0.000000 & -9.824806 & 0.729719 \\ 58 & 1 & 0 & 0.000000 & -8.618647 & 2.497987 \\ 59 & 6 & 0 & 0.000000 & -11.090345 & -1.411798 \\ 60 & 6 & 0 & 0.000000 & -11.090345 & 1.411798 \\ 61 & 6 & 0 & 0.000000 & -12.264759 & 0.717717 \\ 62 & 1 & 0 & 0.000000 & -11.088911 & 2.499305 \\ 63 & 6 & 0 & 0.000000 & -12.264759 & -0.717717 \\ 64 & 1 & 0 & 0.000000 & -11.088911 & -2.499305 \\ 65 & 1 & 0 & 0.000000 & -13.213111 & 1.248177 \\ 66 & 1 & 0 & 0.000000 & -13.213111 & -1.248177\end{array}$

\section{Decacene singlet UB3LYP}

$C, 0,11.103849934,-0.000006397,-1.4075488352$

$C, 0,9.8482329833,-0.0000037696,-0.7243027341$

$\mathrm{H}, 0,8.6272697387,0.0000020926,-2.4941016279$

C, $0,8.6263677166,0.0000005993,-1.4058843711$

C, $0,8.6263676238,-0.0000036775,1.4058844501$

C, $0,7.3946180647,0.0000026581,-0.7274228571$

C, $0,9.8482329425,-0.0000061325,0.72430291$

C, $0,7.3946180247,0.000000621,0.7274228396$

C, $0,6.1576321156,0.0000059875,-1.408260264$

C, $0,11.1038498443,-0.0000111909,1.4075490989$

$\mathrm{H}, 0,6.1587998749,0.0000009591,2.4963326892$

$\mathrm{H}, 0,8.6272695519,-0.0000054802,2.4941017092$

$C, 0,4.9338758837,0.0000071459,-0.7303566405$

$\mathrm{H}, 0,6.1588000305,0.0000071141,-2.4963327903$

C, $0,3.6932395082,0.0000089187,-1.4092303953$

C, $0,4.9338758578,0.0000055872,0.7303564526$

$\mathrm{H}, 0,3.6938074894,0.0000053632,2.4972976651$

C, $0,6.1576320386,0.0000024992,1.40826016$

C, $0,2.4679279427,0.0000089777,-0.7316636535$

$\mathrm{H}, 0,3.693807522,0.0000095707,-2.4972978959$

$C, 0,1.230896234,0.0000090884,-1.4093253413$

C, $0,2.4679279379,0.0000080632,0.7316633907$

C, $0,1.2308962443,0.0000080934,1.4093250727$

C, $0,3.6932394775,0.0000065209,1.4092301653$

C, $0,12.2857045263,-0.0000112285,-0.7131222055$

$\mathrm{H}, 0,11.1017963834,-0.0000046216,-2.4950774513$

C, $0,12.2857044848,-0.0000137323,0.7131225505$

$\mathrm{H}, 0,11.1017962114,-0.0000131335,2.4950777168$

$\mathrm{H}, 0,13.232152148,-0.0000133072,-1.2469066051$

$\mathrm{H}, 0,13.2321520785,-0.0000177737,1.2469070001$

$\mathrm{C}, 0,-0.0000000358,0.0000082849,-0.731886028$

$\mathrm{H}, 0,1.2309407242,0.0000092257,-2.4974032526$

C, $0,-0.0000000141,0.0000082006,0.7318857609$

$\mathrm{H}, 0,1.2309407415,0.0000074953,2.4974029821$

C, $0,-1.2308962529,0.0000076919,1.4093250917$

C, $0,-1.2308962965,0.0000070699,-1.4093253334$

$\mathrm{H}, 0,-1.2309409025,0.0000065822,-2.4974032431$

C, $0,-2.4679279916,0.0000059283,-0.7316636277$

$\mathrm{H}, 0,-1.2309408652,0.0000077165,2.4974030016$

C, $0,-2.4679279542,0.000006663,0.7316634179$

C, $0,-3.6932395448,0.000003758,-1.4092303631$

C, $0,-3.6932394739,0.000005869,1.4092302163$

$\mathrm{H}, 0,-3.6938076762,0.0000027295,-2.4972978613$

$\mathrm{C}, 0,-4.9338758932,0.0000025153,-0.7303565893$

$\mathrm{H}, 0,-3.6938075615,0.0000064427,2.4972977161$

C, $0,-4.9338758567,0.0000039853,0.7303565112$

C, $0,-6.1576321185,-0.0000003628,-1.4082602144$ 
C, $0,-6.1576320438,0.0000030933,1.4082602021$

$\mathrm{H}, 0,-6.158800141,-0.0000019126,-2.4963327377$

C, $0,-7.3946180421,-0.0000016039,-0.7274228171$

$\mathrm{H}, 0,-6.1588000349,0.0000042561,2.496332726$

$C, 0,-7.3946180015,0.0000004705,0.7274228674$

$C, 0,-8.6263675826,-0.000000532,1.4058844649$

C, $0,-8.6263676552,-0.0000049952,-1.405884361$

$\mathrm{C}, 0,-9.8482328741,-0.0000037313,0.7243028971$

$\mathrm{H}, 0,-8.6272695873,0.0000010594,2.4941017255$

C, $0,-9.8482329237,-0.0000062332,-0.7243027489$

$\mathrm{H}, \mathrm{O},-8.6272696532,-0.0000068716,-2.4941016197$

C, $0,-11.1038497569,-0.0000048442,1.4075490802$

C, $0,-11.1038498431,-0.0000099762,-1.4075488685$

C, $0,-12.2857044276,-0.0000110149,-0.7131222392$

$\mathrm{H}, 0,-11.1017963059,-0.0000120823,-2.4950774888$

C, $0,-12.2857043756,-0.000008304,0.7131225105$

$\mathrm{H}, 0,-11.1017961902,-0.0000029491,2.4950777005$

$\mathrm{H}, 0,-13.232152039,-0.0000139411,-1.2469066231$

$\mathrm{H}, 0,-13.2321519644,-0.0000090986,1.2469069422$

\section{Decacene triplet}

\begin{tabular}{|c|c|c|c|c|c|}
\hline $\begin{array}{l}\text { Center } \\
\text { Number }\end{array}$ & $\begin{array}{l}\text { Atomic } \\
\text { Number }\end{array}$ & $\begin{array}{r}\text { Atomic } \\
\text { Type }\end{array}$ & $\begin{array}{l}\text { Coord } \\
\mathrm{X}\end{array}$ & $\begin{array}{c}\text { nates (Angs } \\
y\end{array}$ & roms ) \\
\hline 1 & 6 & 0 & 0.000000 & 11.100510 & 1.408171 \\
\hline 2 & 6 & 0 & 0.000000 & 9.843216 & 0.724769 \\
\hline 3 & 1 & 0 & 0.000000 & 8.624028 & 2.493981 \\
\hline 4 & 6 & 0 & 0.000000 & 8.623551 & 1.405756 \\
\hline 5 & 6 & 0 & 0.000000 & 8.623551 & -1.405756 \\
\hline 6 & 6 & 0 & 0.000000 & 7.391960 & 0.726827 \\
\hline 7 & 6 & 0 & 0.000000 & 9.843216 & -0.724769 \\
\hline 8 & 6 & 0 & 0.000000 & 7.391960 & -0.726827 \\
\hline 9 & 6 & 0 & 0.000000 & 6.153900 & 1.407388 \\
\hline 10 & 6 & 0 & 0.000000 & 11.100510 & -1.408171 \\
\hline 11 & 1 & 0 & 0.000000 & 6.155036 & -2.495506 \\
\hline 12 & 1 & 0 & 0.000000 & 8.624028 & -2.493981 \\
\hline 13 & 6 & 0 & 0.000000 & 4.935773 & 0.729914 \\
\hline 14 & 1 & 0 & 0.000000 & 6.155036 & 2.495506 \\
\hline 15 & 6 & 0 & 0.000000 & 3.688488 & 1.408966 \\
\hline 16 & 6 & 0 & 0.000000 & 4.935773 & -0.729914 \\
\hline 17 & 1 & 0 & 0.000000 & 3.689893 & -2.497071 \\
\hline 18 & 6 & 0 & 0.000000 & 6.153900 & -1.407388 \\
\hline 19 & 6 & 0 & 0.000000 & 2.471753 & 0.733635 \\
\hline 20 & 1 & 0 & 0.000000 & 3.689893 & 2.497071 \\
\hline 21 & 6 & 0 & 0.000000 & 1.228434 & 1.410391 \\
\hline 22 & 6 & 0 & 0.000000 & 2.471753 & -0.733635 \\
\hline 23 & 6 & 0 & 0.000000 & 1.228434 & -1.410391 \\
\hline 24 & 6 & 0 & 0.000000 & 3.688488 & -1.408966 \\
\hline 25 & 6 & 0 & 0.000000 & 12.280713 & 0.713968 \\
\hline 26 & 1 & 0 & 0.000000 & 11.098271 & 2.495687 \\
\hline 27 & 6 & 0 & 0.000000 & 12.280713 & -0.713968 \\
\hline 28 & 1 & 0 & 0.000000 & 11.098271 & -2.495687 \\
\hline 29 & 1 & 0 & 0.000000 & 13.227611 & 1.246970 \\
\hline 30 & 1 & 0 & 0.000000 & 13.227611 & -1.246970 \\
\hline 31 & 6 & 0 & 0.000000 & 0.000000 & 0.735678 \\
\hline 32 & 1 & 0 & 0.000000 & 1.229183 & 2.498466 \\
\hline 33 & 6 & 0 & 0.000000 & 0.000000 & -0.735678 \\
\hline 34 & 1 & 0 & 0.000000 & 1.229183 & -2.498466 \\
\hline 35 & 6 & 0 & 0.000000 & -1.228434 & -1.410391 \\
\hline 36 & 6 & 0 & 0.000000 & -1.228434 & 1.410391 \\
\hline 37 & 1 & 0 & 0.000000 & -1.229183 & 2.498466 \\
\hline
\end{tabular}




$\begin{array}{rrrrrr}38 & 6 & 0 & 0.000000 & -2.471753 & 0.733635 \\ 39 & 1 & 0 & 0.00000 & -1.229183 & -2.498466 \\ 40 & 6 & 0 & 0.000000 & -2.471753 & -0.733635 \\ 41 & 6 & 0 & 0.000000 & -3.688488 & 1.408966 \\ 42 & 6 & 0 & 0.000000 & -3.688488 & -1.408966 \\ 43 & 1 & 0 & 0.00000 & -3.689893 & 2.497071 \\ 44 & 6 & 0 & 0.000000 & -4.935773 & 0.729914 \\ 45 & 1 & 0 & 0.000000 & -3.689893 & -2.497071 \\ 46 & 6 & 0 & 0.000000 & -4.935773 & -0.729914 \\ 47 & 6 & 0 & 0.000000 & -6.153900 & 1.407388 \\ 48 & 6 & 0 & 0.00000 & -6.153900 & -1.407388 \\ 49 & 1 & 0 & 0.000000 & -6.155036 & 2.495506 \\ 50 & 6 & 0 & 0.000000 & -7.391960 & 0.726827 \\ 51 & 1 & 0 & 0.000000 & -6.155036 & -2.495506 \\ 52 & 6 & 0 & 0.000000 & -7.391960 & -0.726827 \\ 53 & 6 & 0 & 0.000000 & -8.623551 & -1.405756 \\ 54 & 6 & 0 & 0.000000 & -8.623551 & 1.405756 \\ 55 & 6 & 0 & 0.000000 & -9.843216 & -0.724769 \\ 56 & 1 & 0 & 0.000000 & -8.624028 & -2.493981 \\ 57 & 6 & 0 & 0.000000 & -9.843216 & 0.724769 \\ 58 & 1 & 0 & 0.000000 & -8.624028 & 2.493981 \\ 59 & 6 & 0 & 0.000000 & -11.100510 & -1.408171 \\ 60 & 6 & 0 & 0.000000 & -11.100510 & 1.408171 \\ 61 & 6 & 0 & 0.000000 & -12.280713 & 0.713968 \\ 62 & 1 & 0 & 0.000000 & -11.098271 & 2.495687 \\ 63 & 6 & 0 & 0.000000 & -12.280713 & -0.713968 \\ 64 & 1 & 0 & 0.000000 & -11.098271 & -2.495687 \\ 65 & 1 & 0 & 0.000000 & -13.227611 & 1.246970 \\ 66 & 1 & 0 & 0.000000 & -13.227611 & -1.246970\end{array}$

